SELECCIONES MATEMÁTICAS
Universidad Nacional de Trujillo
ISSN: 2411-1783 (Online)
Vol. 06(01): $49-65(2019)$

\title{
Existencia y regularidad de solución de la ecuación del calor en espacios de Sobolev periódico.
}

\section{Existence and regularity of solution of the heat equation in periodic Sobolev spaces.}

\author{
Yolanda Santiago Ayala * and Santiago Rojas Romero ${ }^{\dagger}$ \\ Received, Feb. 19, 2019 \\ Accepted, Jun. 04, 2019
}

DOI: http://dx.doi.org/10.17268/sel.mat.2019.01.08

\begin{abstract}
Resumen
En este artículo probamos que el problema de Cauchy asociado a la ecuación del calor en espacios de Sobolev periódico está bien colocado. Hacemos esto en un modo intuitivo usando la teoría de Fourier y en una versión elegante usando la teoría de semigrupos, inspirados en los trabajos de Iorio [1] y Santiago and Rojas [3]. También, estudiamos la relación entre el dato inicial y la diferenciabilidad de la solución. Finalmente, estudiamos el correspondiente problema no homogéneo y probamos que está localmente bien colocado y más aún obtenemos la dependencia continua de la solución respecto al dato inicial y a la no homogeneidad.
\end{abstract}

Palabras clave. Teoría de semigrupos, Ecuación del calor, Ecuación no homogénea, Espacios de Sobolev periódico, Teoría de Fourier.

Abstract
In this article we prove that the Cauchy problem associated to the heat equation in periodic Sobolev spaces is well posed. We do this in an intuitive way using Fourier theory and in a fine version using Semigroups theory, inspired by works Iorio [1] and Santiago and Rojas [3]. Also, we study the relationship between the initial data and differentiability of the solution.

Finally, we study the corresponding nonhomogeneous problem and prove it is locally well posed and even more we obtain the continuous dependence of the solution with respect to the initial data and the non homogeneity.

Keywords. Semigroups theory, Heat equation, nonhomogeneous equation, Periodic Sobolev spaces, Fourier theory.

1. Introducción. Sea la ecuación del calor propuesto por Fourier (1807)

$$
u_{t}-\alpha^{2} u_{x x}=0 .
$$

Sabemos que la ecuación (1.1) es de tipo parabólica y de su importancia en las Ecuaciones Diferenciales Parciales. En la ecuación (1.1), $\alpha^{2}$ es una constante conocida como el coeficiente de difusión térmica, en este trabajo consideraremos $\alpha^{2}=1$, y como dato inicial consideramos $u(0)=\phi \in H_{p e r}^{s}$, donde $s$ es un número real y denotamos por $H_{p e r}^{s}$ al espacio de Sobolev periódico de orden $s$. Dicha ecuación describe la distribución del calor (o variación de temperatura) en una región a través del tiempo.

La ecuación que estudiamos está asociada a fenómenos de difusión, como el flujo del calor en un medio conductivo, citamos a Rubinstein [4].

Sabemos que la ecuación del calor gobierna la difusión de partículas o la propagación del potencial de acción en células neuronales y que puede utilizarse para modelar algunos problemas en finanzas, como por ejemplo en los

*ID ORCID: https://orcid.org/0000-0003-2516-0871, Facultad de Ciencias Matemáticas, Universidad Nacional Mayor de San Marcos, Av. Venezuela S/N Lima 01, Lima-Perú (ysantiagoa@unmsm.edu . pe).

$\dagger$ ID ORCID: https://orcid.org/0000-0002-5354-8059, Facultad de Ciencias Matemáticas, Universidad Nacional Mayor de San Marcos, Av. Venezuela S/N Lima 01, Lima-Perú (srojasr@unmsm.edu.pe),

This work is licensed under the Creative Commons Attribution-NoComercial-ShareAlike 4.0. 
procesos de Black-Scholes o Ornstein-Uhlenbeck, citamos por ejemplo [5].

Sabemos también, que la ecuación de Burgers $u_{t}-u_{x x}+u u_{x}=0$ se puede transformar en la ecuación del calor vía la transformación de Cole-Hopf, de ahí que se enfatiza el estudio de esta ecuación, para esto podemos citar Iorio [1].

Citamos Iorio [1], donde encontramos trabajos relacionados al modelo (1.1) y Santiago and Rojas [3] de donde nos motivamos siguiendo las ideas ahí plasmadas. Probaremos la existencia y unicidad de solución de (1.1), así como la dependencia continua de la solución respecto al dato inicial. Luego introduciremos una familia de operadores para reescribir nuestro resultado en una versión más elegante. Haremos análisis de diferenciabilidad versus dato inicial del problema y finalmente probaremos que el modelo no homogéneo de (1.1) está localmente bien colocado.

Nuestro artículo está organizado como sigue. En la sección 2, indicamos la metodología usada y citamos la referencia usada para los resultados preliminares que se puedan necesitar. En la sección 3, probamos que el problema de Cauchy asociado a la ecuación del calor homogéneo está bien colocado. En la sección 4, hacemos el análisis de la diferenciabilidad de la solución versus dato inicial. En la sección 5, probamos que el problema de Cauchy asociado a la ecuación del calor no homogéneo esta localmente bien colocado y además obtenemos la dependencia continua de la solución respecto al dato inicial y a la no homogeneidad. Finalmente, en la sección 6, damos las conclusiones de nuestro estudio.

2. Metodología. Como marco teórico, en este trabajo usamos fuertemente los siguientes tópicos: Teoría de Fourier en espacios de Sobolev periódico, análisis armónico, teoría de semigrupos de clase $C_{o}$ y familias de operadores fuertemente continuas. Como referencia en la revisión de algunos resultados previos que usaremos, citamos a Iorio [1], Santiago et al [2] y Santiago and Rojas [3].

Toda esta teoría la usamos en el análisis de existencia y buena colocación del problema de Cauchy para la ecuación del calor, realizando una serie de cálculos y aproximaciones en el desarrollo del trabajo.

3. Existencia de solución de la ecuación del Calor. En esta sección, empezamos probando que existe solución de la ecuación del calor homogéneo en espacios de Sobolev periódico, usando la teoría de Fourier.

Teorema 1. Sea s un número real fijo y el problema

$$
\begin{array}{l|l}
\left(P_{1}\right) & \begin{array}{l}
u \in C\left([0,+\infty), H_{p e r}^{s}\right) \\
\partial_{t} u-\partial_{x}^{2} u=0 \in H_{p e r}^{s-2} \\
u(0)=\phi \in H_{p e r}^{s}
\end{array}
\end{array}
$$

entonces $\left(P_{1}\right)$ está globalmente bien colocado i.e. $\exists ! u \in C\left([0, \infty), H_{p e r}^{s}\right)$ satisfaciendo la ecuación $\left(P_{1}\right)$, de modo que la aplicación $: \phi \rightarrow u$, que asigna a cada dato inicial $\phi$ la solución u del PVI $\left(P_{1}\right)$, es continua. Además, $u \in C\left([0, \infty), H_{\text {per }}^{s}\right) \cap C^{1}\left([0, \infty), H_{\text {per }}^{s-2}\right)$ y la solución u satisface la regularidad:

$$
u(t) \in H^{\infty}, \forall t>0
$$

con $\|u(t)\|_{r} \leq C\|\phi\|_{s}, \forall r \in \mathbb{R}$ y $t>0$, donde $H^{\infty}$ es la intersección de los espacios $H_{\text {per }}^{r} \forall r \in \mathbb{R}$.

Demostración:La prueba lo hacemos del siguiente modo.

1. Primero obtenemos el candidato a solución. Para conseguir ese candidato tomamos la transformada de Fourier a la ecuación

$$
\partial_{t} u=\partial_{x}^{2} u
$$

y conseguimos

$$
\partial_{t} \hat{u}=(i k)^{2} \hat{u}=-k^{2} \hat{u}
$$

que para cada $k$ es una EDO con dato inicial $\hat{u}(k, 0)=\hat{\phi}(k)$.

Así, planteamos un sistema no acoplado de ecuaciones de primer orden homogéneas

$$
\begin{array}{l|l}
\left(\Omega_{k}\right) & \begin{array}{l}
\hat{u} \in C\left([0,+\infty), l_{s}^{2}(Z)\right) \\
\partial_{t} \hat{u}(k, t)=-k^{2} \hat{u}(k, t) \\
\hat{u}(k, 0)=\hat{\phi}(k) \operatorname{con} \hat{\phi} \in l_{s}^{2}(Z)
\end{array}
\end{array}
$$

$\forall k \in Z$, y conseguimos

$$
\hat{u}(k, t)=e^{-k^{2} t} \hat{\phi}(k),
$$

de donde obtenemos nuestro candidato a solución:

$$
u(t)=\sum_{k=-\infty}^{\infty} \hat{u}(k, t) \phi_{k}=\sum_{k=-\infty}^{\infty} e^{-k^{2} t} \hat{\phi}(k) \phi_{k},
$$


aquí estamos denotando $\phi_{k}(x)=e^{i k x}$.

2. En segundo lugar, probaremos que:

$$
u(t) \in H_{p e r}^{s} \quad \text { y }\|u(t)\|_{s} \leq\|\phi\|_{s} .
$$

En efecto, sea $t>0, \phi \in H_{\text {per }}^{s}$ y observando que $e^{-2 k^{2} t}<1$, tenemos

$$
\begin{aligned}
\|u(t)\|_{H_{p e r}^{s}}^{2} & =2 \pi \sum_{k=-\infty}^{+\infty}\left(1+k^{2}\right)^{s}\left|e^{-k^{2} t} \hat{\phi}(k)\right|^{2} \\
& =2 \pi \sum_{k=-\infty}^{+\infty}\left(1+k^{2}\right)^{s}|\hat{\phi}(k)|^{2} e^{-2 k^{2} t} \\
& \leq 2 \pi \sum_{k=-\infty}^{+\infty}\left(1+k^{2}\right)^{s}|\hat{\phi}(k)|^{2}<\infty \\
& =\|\phi\|_{H_{\text {per }}^{s}}^{2} .
\end{aligned}
$$

Obviamente también se cumple (3.2) para $t=0$.

3. Ahora, probaremos que $u(\cdot)$ es continua en $[0,+\infty)$.

Sea $t^{\prime} \in[0, \infty)$,

$$
\begin{aligned}
\| u(t)- & u\left(t^{\prime}\right) \|_{H_{p e r}^{s}}^{2} \\
& =2 \pi \sum_{k=-\infty}^{+\infty}\left(1+k^{2}\right)^{s}\left|\left(e^{-k^{2} t}-e^{-k^{2} t^{\prime}}\right) \hat{\phi}(k)\right|^{2} \\
& =2 \pi \sum_{k=-\infty}^{+\infty}\left(1+k^{2}\right)^{s}|\hat{\phi}(k)|^{2}|\underbrace{\left(e^{-k^{2} t}-e^{-k^{2} t^{\prime}}\right)}_{H(t):=}|^{2} .
\end{aligned}
$$

Se observa que $\operatorname{lím}_{t \rightarrow t^{\prime}} H(t)=0$. Ahora, necesitamos de la convergencia uniforme de la serie para el intercambio de límites. Para esto, tomamos el k-ésimo término de la serie y lo mayoramos por una serie convergente, i.e.

$$
I_{k, t}:=2 \pi\left(1+k^{2}\right)^{s}|\hat{\phi}(k)|^{2}|\underbrace{\left(e^{-k^{2} t}-e^{-k^{2} t^{\prime}}\right)}|^{2} \leq 8 \pi\left(1+k^{2}\right)^{s}|\hat{\phi}(k)|^{2},
$$

donde hemos usado la desigualdad triangular (propiedad de la norma) y la desigualdad $e^{-\theta} \leq 1$ siempre que $\theta \geq 0$.

Así,

$$
\sum_{k=-\infty}^{+\infty} I_{k, t} \leq 4\|\phi\|_{H_{p e r}^{s}}^{2}<\infty
$$

y usando el Teorema del M-Test de Weierstrass tenemos que la serie converge uniformemente. Luego está permitido el intercambio de límite, esto es

$$
\lim _{t \rightarrow t^{\prime}}\left\|u(t)-u\left(t^{\prime}\right)\right\|_{H_{p e r}^{s}}^{2}=\sum_{k=-\infty}^{+\infty} \lim _{t \rightarrow t^{\prime}} I_{k, t}=0
$$

y de ahí concluimos

$$
\lim _{t \rightarrow t^{\prime}}\left\|u(t)-u\left(t^{\prime}\right)\right\|_{H_{p e r}^{s}}=0
$$

4. Probaremos que $\partial_{t} u=\partial_{x}^{2} u$ en $H_{p e r}^{s-2}$, esto es,

$$
\left\|\frac{u(t+h)-u(t)}{h}-\partial_{x}^{2} u\right\|_{H_{p e r}^{s-2}} \longrightarrow 0 \quad \text { cuando } h \rightarrow 0 .
$$


En efecto,

$$
\begin{aligned}
& \left\|\frac{u(t+h)-u(t)}{h}-\partial_{x}^{2} u\right\|_{H_{\text {per }}^{s-2}}^{2} \\
& =2 \pi \sum_{k=-\infty}^{+\infty}\left(1+k^{2}\right)^{s-2}|\hat{\phi}(k)|^{2}\left|\frac{e^{-k^{2}(t+h)}-e^{-k^{2} t}}{h}-(i k)^{2} e^{-k^{2} t}\right|^{2} \\
& =2 \pi \sum_{k=-\infty}^{+\infty}\left(1+k^{2}\right)^{s-2}|\hat{\phi}(k)|^{2}|e^{-k^{2} t} \cdot \underbrace{\left\{\frac{e^{-k^{2} h}-1}{h}+k^{2}\right\}}_{M(h):=}|^{2} .
\end{aligned}
$$

Usando L'Hospital tenemos que $M(h) \longrightarrow 0$ cuando $h \rightarrow 0$.

Ahora, necesitamos la convergencia uniforme de la serie para habilitar el intercambio de límites. Para ello procedemos mayorando el k-ésimo término de la serie. Previamente observamos para $h>0$ :

$$
\frac{e^{-k^{2} h}-1}{h}=\int_{0}^{h} \frac{1}{h} \frac{\partial}{\partial s}\left\{e^{-k^{2} s}\right\} d s=\int_{0}^{h} \frac{1}{h}\left[-k^{2}\right] e^{-k^{2} s} d s
$$

y tomando norma tenemos

$$
\left|\frac{e^{-k^{2} h}-1}{h}\right| \leq \frac{1}{h} k^{2} \int_{0}^{h}\left|e^{-k^{2} s}\right| \leq \frac{1}{h}|k|^{2} \cdot h=|k|^{2} .
$$

O también se puede proceder usando el Teorema del valor medio, aplicado a la función $f(t)=e^{-t k^{2}}$ en el intervalo $[0, h]$.

Usando la desigualdad (3.5) procedemos a mayorar $[M(h)]^{2}$ como sigue

$$
[M(h)]^{2} \leq\left\{2|k|^{2}\right\}^{2} \leq 4\left\{1+|k|^{2}\right\}^{2}
$$

La desigualdad (3.6) también es válido para el caso $t=0$, donde sólo usamos (3.5).

Ahora, pasamos a mayorar el k-ésimo término de la serie, donde usamos la estimativa (3.6),

$$
\begin{aligned}
\left(1+k^{2}\right)^{s-2}|\hat{\phi}(k)|^{2} e^{-2 k^{2} t}[M(h)]^{2} & \leq\left(1+k^{2}\right)^{s-2}|\hat{\phi}(k)|^{2}[M(h)]^{2} \\
& \leq\left(1+k^{2}\right)^{s-2}|\hat{\phi}(k)|^{2} 4\left(1+|k|^{2}\right)^{2} \\
& =4\left(1+k^{2}\right)^{s}|\hat{\phi}(k)|^{2}
\end{aligned}
$$

y también sabemos que la serie $2 \pi \sum_{k=-\infty}^{+\infty}\left(1+k^{2}\right)^{s}|\hat{\phi}(k)|^{2}=\|\phi\|_{H_{p e r}^{s}}^{2}<\infty$ desde que $\phi \in H_{p e r}^{s}$. Usando el Teorema M-Test de Weierstrass tenemos que la serie (3.4) converge uniformemente y por lo tanto es posible intercambiar límites y obtener lo que se quería mostrar, i.e.

$$
\left\|\frac{u(t+h)-u(t)}{h}-\partial_{x}^{2} u\right\|_{H_{p e r}^{s-2}}^{2} \longrightarrow 0 \quad \text { cuando } h \rightarrow 0
$$

y esto implica lo que se quería probar.

5. Probaremos la dependencia continua de la solución respecto a los datos iniciales, i.e. sean $\phi$ y $\widetilde{\phi}$ datos próximos en $H_{\text {per }}^{s}$, entonces sus correspondientes soluciones $u$ y $\widetilde{u}$, respectivamente, también están próximos en el espacio solución. 
Sea $t>0$,

$$
\begin{aligned}
\|u(t)-\widetilde{u}(t)\|_{H_{p e r}^{s}}^{2} & =2 \pi \sum_{k=-\infty}^{+\infty}\left|e^{-k^{2} t}(\widehat{\phi}(k)-\widehat{\widetilde{\phi}}(k))\right|^{2}\left(1+k^{2}\right)^{s} \\
& =2 \pi \sum_{k=-\infty}^{+\infty} \underbrace{e^{-2 k^{2} t}}_{\leq 1}|\widehat{\phi}(k)-\widehat{\widetilde{\phi}}(k)|^{2}\left(1+k^{2}\right)^{s} \\
& \leq 2 \pi \sum_{k=-\infty}^{+\infty}\left(1+k^{2}\right)^{s}|\widehat{\phi}(k)-\widehat{\widetilde{\phi}}(k)|^{2} \\
& =\|\phi-\widetilde{\phi}\|_{H_{p e r}^{s}}^{2} .
\end{aligned}
$$

Tomando supremo sobre $(0,+\infty)$ tenemos

$$
\sup _{t \in(0,+\infty)}\|u(t)-\widetilde{u}(t)\|_{H_{p e r}^{s}} \leq\|\phi-\widetilde{\phi}\|_{H_{p e r}^{s}} .
$$

Así,

$$
\sup _{t \in[0,+\infty)}\|u(t)-\widetilde{u}(t)\|_{H_{p e r}^{s}}=\|\phi-\widetilde{\phi}\|_{H_{p e r}^{s}} .
$$

De aquí tenemos que si $\phi \rightarrow \widetilde{\phi}$ entonces $u \rightarrow \widetilde{u}$.

6. Unicidad de Solución.- La desigualdad (3.7) o igualdad (3.8) nos permitirá mostrar que la solución es única. En efecto, sea $\phi \in H_{\text {per }}^{s}$ y supongamos que existan $u$ y $\widetilde{u}$ dos soluciones, entonces usando (3.7) o (3.8) tenemos,

$$
\|u(t)-\widetilde{u}(t)\|_{H_{p e r}^{s}} \leq \sup _{t \in[0,+\infty)}\|u(t)-\widetilde{u}(t)\|_{H_{p e r}^{s}}=\|\phi-\phi\|_{H_{p e r}^{s}}=0
$$

de donde concluimos que $u=\widetilde{u}$.

Así, el problema $\left(P_{1}\right)$ está bien colocado y su única solución que depende continuamente del dato inicial es

$$
u(x, t)=\sum_{k=-\infty}^{+\infty} e^{-k^{2} t} \hat{\phi}(k) e^{i k x}
$$

7. Probaremos que $\partial_{t} u(\cdot)$ es continua en $[0,+\infty)$. En efecto, como $\partial_{t} u(t)=\partial_{x}^{2} u(t)$ en $H_{\text {per }}^{s-2}$ tenemos

$$
\begin{aligned}
\left\|\partial_{t} u(t)-\partial_{t} u\left(t^{\prime}\right)\right\|_{H_{\text {per }}^{s-2}} & =\left\|\partial_{x}^{2} u(t)-\partial_{x}^{2} u\left(t^{\prime}\right)\right\|_{s-2} \\
& \leq\left\|u(t)-u\left(t^{\prime}\right)\right\|_{s} \rightarrow 0 \text { cuando } t \rightarrow t^{\prime} .
\end{aligned}
$$

Luego $\partial_{t} u(\cdot)$ es continua en $t^{\prime}$.

Análogo, usando la inmersion $H_{\text {per }}^{s-2} \subset H_{\text {per }}^{r-2}$ para $r \leq s$, obtenemos

$$
\begin{aligned}
\left\|\partial_{t} u(t)-\partial_{t} u\left(t^{\prime}\right)\right\|_{H_{\text {per }}^{r-2}} & =\left\|\partial_{x}^{2} u(t)-\partial_{x}^{2} u\left(t^{\prime}\right)\right\|_{r-2} \\
& \leq\left\|\partial_{x}^{2} u(t)-\partial_{x}^{2} u\left(t^{\prime}\right)\right\|_{s-2} \\
& \leq\left\|u(t)-u\left(t^{\prime}\right)\right\|_{s} \rightarrow 0 \text { cuando } t \rightarrow t^{\prime}
\end{aligned}
$$

8. Sea $t>0$, para $r>s$ tenemos

$$
\begin{aligned}
\|u(t)\|_{r}^{2} & =2 \pi \sum_{k=-\infty}^{+\infty}\left(1+k^{2}\right)^{r}|\widehat{\phi}(k)|^{2}\left|e^{-k^{2} t}\right|^{2} \\
& =2 \pi \sum_{k=-\infty}^{+\infty}\left(1+k^{2}\right)^{s}|\widehat{\phi}(k)|^{2} \underbrace{e^{-2 k^{2} t} \cdot\left(1+k^{2}\right)^{r-s}}_{G(k, t):=} \\
& \leq C^{*} 2 \pi \sum_{k=-\infty}^{+\infty}\left(1+k^{2}\right)^{s}|\widehat{\phi}(k)|^{2}<\infty \\
& =C^{*}\|\phi\|_{s}^{2}
\end{aligned}
$$


donde $|G(k, t)| \leq C^{*}, \forall k \in Z, t>0$. Así,

$$
u(t) \in H_{p e r}^{r}, \forall r \in(s,+\infty)
$$

El caso $r=s$ ya lo hemos probado en el item 2.

9. Ahora, consideramos el caso $r<s$. Como $r<s$ entonces $H_{p e r}^{s} \subset H_{p e r}^{r}$ y desde que el dato inicial $\phi \in H_{p e r}^{s}$, entonces $\phi \in H_{p e r}^{r}$ y satisface

$$
\|\phi\|_{r} \leq\|\phi\|_{s}
$$

De (3.2) y usando (3.10) tenemos que

$$
\|u(t)\|_{r}^{2} \leq\|\phi\|_{r}^{2} \leq\|\phi\|_{s}^{2}<\infty .
$$

Es decir,

$$
u(t) \in H_{p e r}^{r}, \forall r \in(-\infty, s)
$$

Finalmente, de (3.9), (3.2) y (3.11) concluimos que para $t>0$

$$
u(t) \in H_{p e r}^{r}, \forall r \in \mathbb{R},
$$

y existe $C:=\operatorname{máx}\left\{1, \sqrt{C^{*}}\right\}$ tal que $\|u(t)\|_{r} \leq C\|\phi\|_{s} \forall r \in \mathbb{R}$ y $\forall t>0$.

En consecuencia tenemos los siguientes resultados

Corolario 1. La única solución de $\left(P_{1}\right)$ es

$$
u(x, t)=\sum_{k=-\infty}^{+\infty} e^{-k^{2} t} \hat{\phi}(k) e^{i k x} .
$$

Corolario 2. La solución u de la ecuación homogénea $\left(P_{1}\right)$ satisface

$$
\begin{aligned}
\|u(t)\|_{s} & \leq\|\phi\|_{s}, \quad \forall t>0 \\
\left\|\partial_{t} u(t)\right\|_{s-2} & \leq\|\phi\|_{s}, \quad \forall t>0 .
\end{aligned}
$$

Demostración: De (3.2) obtenemos (3.12). Por otro lado, sabemos que en $H_{\text {per }}^{s-2}$ se tiene $\partial_{t} u(t)=\partial_{x}^{2} u(t)$, entonces $\left\|\partial_{t} u(t)\right\|_{s-2}=\left\|\partial_{x}^{2} u(t)\right\|_{s-2} \leq\|u(t)\|_{s} \leq\|\phi\|_{s}$.

Ahora, introduciremos una familia de operadores que verificaran las condiciones de ser un semigrupo de contracción de clase $C_{0}$.

Teorema 2. Sea $s \in \mathbb{R}$ y la aplicación

$$
\begin{aligned}
S:[0,+\infty) & \rightarrow L\left(H_{p e r}^{s}\right) \\
t & \rightarrow S(t)
\end{aligned}
$$

tal que $S(t)=e^{\partial_{x}^{2} t}$, i.e. aplica $S(t) \phi=\left\{e^{-k^{2} t} \hat{\phi}(k)\right\}^{\vee}, \forall \phi \in H_{\text {per }}^{s}$.

Entonces $\{S(t)\}_{t \geq 0}$ es un semigrupo de clase $C_{o}$ de contracción en $H_{p e r}^{s}$.

Además, se verifican los siguientes enunciados:

1. $S(\cdot) \phi \in C\left([0, \infty), H_{\text {per }}^{s}\right)$.

2. La aplicación $\phi \rightarrow S(\cdot) \phi$ es continua $y \forall \varphi_{1}, \varphi_{2} \in H_{\text {per }}^{s}$ se satisface:

$$
\begin{aligned}
\left\|S(t) \varphi_{1}-S(t) \varphi_{2}\right\|_{H_{p e r}^{s}} & \leq\left\|\varphi_{1}-\varphi_{2}\right\|_{H_{p e r}^{s}}, \forall t \geq 0 \\
\sup _{t>0}\left\|S(t) \varphi_{1}-S(t) \varphi_{2}\right\|_{H_{p e r}^{s}} & \leq\left\|\varphi_{1}-\varphi_{2}\right\|_{H_{p e r}^{s}} .
\end{aligned}
$$

3. $S(t) \in L\left(H_{\text {per }}^{s}, H_{\text {per }}^{r}\right) \forall t>0, \forall r \in \mathbb{R}$ y satisface:

$$
\|S(t) \phi\|_{r} \leq c\|\phi\|_{s}, \forall \phi \in H_{p e r}^{s}, \forall r \in \mathbb{R}, t>0
$$

4. En particular vale $\|S(t) \phi\|_{s} \leq\|\phi\|_{s}$ y además $\left\|\partial_{t} S(t) \phi\right\|_{s-2} \leq\|\phi\|_{s}$. 
Demostración:Primero observamos que $S(0) \phi=\phi, \forall \phi \in H_{\text {per }}^{s}$, así $S(0)=I$. De la linealidad de la transformada de Fourier y de su inversa tenemos que $S(t)$ es lineal.

Si $\phi \in H_{\text {per }}^{s}$ probaremos que $S(t) \phi \in H_{\text {per }}^{s}$ y $\|S(t) \phi\|_{s} \leq\|\phi\|_{s}$, i.e. $\|S(t)\| \leq 1$. En efecto, análogo a (3.3) tenemos

$$
\begin{aligned}
\|S(t) \phi\|_{H_{p e r}^{s}}^{2} & =2 \pi \sum_{k=-\infty}^{+\infty}\left(1+k^{2}\right)^{s}\left|e^{-k^{2} t} \hat{\phi}(k)\right|^{2} \\
& =2 \pi \sum_{k=-\infty}^{+\infty}\left(1+k^{2}\right)^{s}|\hat{\phi}(k)|^{2} e^{-2 k^{2} t} \\
& \leq 2 \pi \sum_{k=-\infty}^{+\infty}\left(1+k^{2}\right)^{s}|\hat{\phi}(k)|^{2}=\|\phi\|_{H_{p e r}^{s}}^{2} \quad<\infty .
\end{aligned}
$$

Luego, $S(t) \phi \in H_{\text {per }}^{s}$ y $\|S(t) \phi\|_{s} \leq\|\phi\|_{s}$, es decir $S(t) \in L\left(H_{\text {per }}^{s}\right)$ con $\|S(t)\| \leq 1$. Ahora probaremos que $S(t+r)=S(t) \circ S(r), \forall t, r \geq 0$.

$$
\begin{aligned}
S(t+r) f(x) & =\sum_{k=-\infty}^{\infty} e^{-k^{2}(t+r)} \hat{f}(k) e^{i k x} \\
& =\sum_{k=-\infty}^{\infty} e^{-k^{2} t} \underbrace{e^{-k^{2} r} \hat{f}(k)}_{\hat{g}(k):=} e^{i k x} \\
& =S(t) g(x)
\end{aligned}
$$

donde $g$ es tal que $\hat{g}(k)=e^{-k^{2} r} \hat{f}(k), \forall k \in Z$. Así,

$$
g(x)=\sum_{k=-\infty}^{\infty} e^{-k^{2} r} \hat{f}(k) e^{i k x}=S(r) f(x) .
$$

Por lo tanto, $S(t+r) f=S(t) \circ S(r) f, \forall t, r \geq 0$.

Ahora probaremos la continuidad de $t \rightarrow S(t) \phi$, esto es

$$
\|S(t+h) \phi-S(t) \phi\|_{H_{p e r}^{s}} \rightarrow 0 \text { cuando } h \rightarrow 0 .
$$

En efecto, usando el item 3 de la prueba del teorema anterior, tenemos

$$
\begin{aligned}
\|S(t+h) \phi-S(t) \phi\|_{H_{p e r}^{s}}^{2} & \\
& =2 \pi \sum_{k=-\infty}^{+\infty}\left(1+k^{2}\right)^{s}\left|\left(e^{-k^{2}(t+h)}-e^{-k^{2} t}\right) \hat{\phi}(k)\right|^{2} \\
& =2 \pi \sum_{k=-\infty}^{+\infty}\left(1+k^{2}\right)^{s}|\hat{\phi}(k)|^{2}|\underbrace{\left(e^{-k^{2}(t+h)}-e^{-k^{2} t}\right)}_{H(t, h):=}|^{2} .
\end{aligned}
$$

Observamos que $\operatorname{lím}_{h \rightarrow 0} H(t, h)=0$.

Ahora, necesitamos de la convergencia uniforme de la serie para el intercambio de límites. Para eso, tomamos el k-ésimo término de la serie y lo mayoramos por una serie convergente, i.e.

$$
I_{k, t, h}:=2 \pi\left(1+k^{2}\right)^{s}|\hat{\phi}(k)|^{2}\left|\left(e^{-k^{2}(t+h)}-e^{-k^{2} t}\right)\right|^{2} \leq 8 \pi\left(1+k^{2}\right)^{s}|\hat{\phi}(k)|^{2},
$$

donde hemos usado la desigualdad triangular (propiedad de la norma) y la desigualdad $e^{-\theta} \leq 1$ siempre que $\theta \geq 0$. Así,

$$
\sum_{k=-\infty}^{+\infty} I_{k, t, h} \leq 4\|\phi\|_{H_{p e r}^{s}}^{2}<\infty,
$$

y usando el Teorema del M-Test de Weierstrass tenemos que la serie en (3.17) converge uniformemente. Luego está permitido el intercambio de límite, esto es,

$$
\lim _{h \rightarrow 0}\|S(t+h) \phi-S(t) \phi\|_{H_{p e r}^{s}}^{2}=\sum_{k=-\infty}^{+\infty} \lim _{h \rightarrow 0} I_{k, t, h}=0
$$


y de ahí concluimos

$$
\lim _{h \rightarrow 0}\|S(t+h) \phi-S(t) \phi\|_{H_{p e r}^{s}}=0
$$

Observación 1. También se verifica

$$
\lim _{t \rightarrow 0^{+}}\|S(t) \phi-\phi\|_{H_{p e r}^{s}}=0 .
$$

Por lo tanto, $\{S(t)\}_{t \geq 0}$ es un semigrupo de clase $C_{o}$ de Contracción en $H_{p e r}^{s}$.

Ahora, sean $\varphi_{1}$ y $\varphi_{2}$ datos próximos en $H_{\text {per }}^{s}$, entonces probaremos que sus correspondientes $S(\cdot) \varphi_{1}$ y $S(\cdot) \varphi_{2}$, respectivamente, también están próximos. Como $\{S(t)\}_{t \geq 0}$ es de contracción, para $t \geq 0$ tenemos

$$
\left\|S(t) \varphi_{1}-S(t) \varphi_{2}\right\|_{H_{p e r}^{s}}=\left\|S(t)\left[\varphi_{1}-\varphi_{2}\right]\right\|_{H_{p e r}^{s}} \leq\left\|\varphi_{1}-\varphi_{2}\right\|_{H_{p e r}^{s}} .
$$

Tomando supremo sobre $(0,+\infty)$ tenemos

$$
\sup _{t \in(0,+\infty)}\left\|S(t) \varphi_{1}-S(t) \varphi_{2}\right\|_{H_{p e r}^{s}} \leq\left\|\varphi_{1}-\varphi_{2}\right\|_{H_{p e r}^{s}} .
$$

De aquí tenemos que si $\varphi_{1} \rightarrow \varphi_{2}$ entonces $S(\cdot) \varphi_{1} \rightarrow S(\cdot) \varphi_{2}$.

Usando la inmersion $H_{p e r}^{s} \subset H_{p e r}^{r}, \forall r \leq s$ y (3.14), obtenemos

$$
\|S(t) \phi\|_{r} \leq\|S(t) \phi\|_{s} \leq\|\phi\|_{s}, \forall r \leq s .
$$

Sabemos que en $H_{p e r}^{s-2}$ vale

$$
\partial_{t} S(t) \phi=\partial_{x}^{2} S(t) \phi, \forall \phi \in H_{p e r}^{s},
$$

cuya prueba es análoga al item 4 de la prueba del Teorema 1.

Usando la igualdad (3.19) obtenemos:

$$
\left\|\partial_{t} S(t) \phi\right\|_{s-2}=\left\|\partial_{x}^{2} S(t) \phi\right\|_{s-2} \leq\|S(t) \phi\|_{s} \leq\|\phi\|_{s} .
$$

A seguir enunciamos el Teorema 1 en función del semigrupo $\{S(t)\}_{t \geq 0}$.

Teorema 3. Sea $s \in \mathbb{R}$ y $\{S(t)\}_{t \geq 0}$ el semigrupo de clase $C_{0}$ del Teorema 2, $S(\cdot) \phi$ es la única solución de

$$
\mid \begin{aligned}
& u \in C\left([0, \infty), H_{p e r}^{s}\right) \\
& u_{t}=A u \text { en } H_{p e r}^{s-2} \\
& u(0)=\phi \in H_{p e r}^{s}
\end{aligned}
$$

en el sentido que

$$
\lim _{h \rightarrow 0}\left\|\frac{S(t+h) \phi-S(t) \phi}{h}-A S(t) \phi\right\|_{H_{p e r}^{s-2}}=0,
$$

donde $A:=\partial_{x}^{2}$, y si $\varphi_{1} \sim \varphi_{2}$ entonces $S(\cdot) \varphi_{1} \sim S(\cdot) \varphi_{2}$.

Además, $S(\cdot) \phi \in C\left([0, \infty), H_{\text {per }}^{s}\right) \cap C^{1}\left([0, \infty), H_{\text {per }}^{s-2}\right)$ y se satisface la siguiente regularidad: $S i \phi \in H_{\text {per }}^{s}$ entonces $S(t) \phi \in H^{\infty} \forall t>0$ y existe una constante $C>0$ tal que $\|S(t) \phi\|_{H_{\text {per }}^{r}} \leq C\|\phi\|_{H_{\text {per }}^{s}} \forall t>0$ y $\forall r \in \Re$.

Demostración:La prueba de (3.20) es análoga al del item 4 de la prueba del Teorema 1. Y la prueba del resto del enunciado también se sigue como la prueba del Teorema 1 y como consecuencia del Teorema 2.

4. Análisis de la diferenciabilidad versus datos iniciales. Con la finalidad de incrementar y enriquecer nuestro estudio nuevamente buscaremos el espacio infinito dimensional donde ocurre la diferenciabilidad y su conexión con los datos iniciales.

Teorema 4. Sea $s \in \mathbb{R}$. Si $t>0$ y u es solución de $\left(P_{1}\right)$ entonces $\forall r \in \mathbb{R}$ vale:

$$
\lim _{h \rightarrow 0}\left\|\frac{u(t+h)-u(t)}{h}-\partial_{x}^{2} u(t)\right\|_{r}=0 .
$$

Esto quiere decir que la diferenciabilidad se dá en $H_{p e r}^{r}, \forall r \in \mathbb{R}$, i.e. $\partial_{t} u(t)=\partial_{x}^{2} u(t)$ en $H_{p e r}^{r}, \forall r \in \mathbb{R}, \forall t>0$. 
Demostración:Sea $t>0, \phi \in H_{p e r}^{s}$

$$
\begin{aligned}
& \left\|\frac{u(t+h)-u(t)}{h}-\partial_{x}^{2} u(t)\right\|_{r}^{2} \\
& =2 \pi \sum_{k=-\infty}^{+\infty}\left(1+k^{2}\right)^{r} e^{-2 k^{2} t}\left|\frac{e^{-k^{2} h}-1}{h}+k^{2}\right|^{2}|\widehat{\phi}(k)|^{2} \\
& =2 \pi \sum_{k=-\infty}^{+\infty}\left(1+k^{2}\right)^{r-s} e^{-2 k^{2} t}|\underbrace{\frac{e^{-k^{2} h}-1}{h}+k^{2}}_{M(h):=}|^{2}|\widehat{\phi}(k)|^{2}\left(1+k^{2}\right)^{s} .
\end{aligned}
$$

Usando L'Hospital tenemos que $M(h) \longrightarrow 0$ cuando $h \rightarrow 0$.

Ahora, necesitamos la convergencia uniforme de la serie para habilitar el intercambio de límites. Para ello procedemos mayorando el k-ésimo término de la serie (4.1). Previamente usando el Teorema del valor medio, aplicado a la función $f(t)=e^{-t k^{2}}$ en el intervalo $[0, h]$, obtenemos para $h>0$ :

$$
\left|\frac{e^{-k^{2} h}-1}{h}\right| \leq \frac{1}{h}|k|^{2} \cdot h=|k|^{2} .
$$

Usando la desigualdad (4.2) procedemos a mayorar $[M(h)]^{2}$ como sigue

$$
[M(h)]^{2} \leq\left\{2|k|^{2}\right\}^{2} \leq 4\left\{1+|k|^{2}\right\}^{2} .
$$

Pasamos a mayorar el k-ésimo término de la serie, donde usamos la estimativa (4.3)

$$
\begin{aligned}
I_{k, t, r} & :=\left(1+k^{2}\right)^{r-s} e^{-2 k^{2} t}|\hat{\phi}(k)|^{2}[M(h)]^{2}\left(1+k^{2}\right)^{s} \\
& \leq \underbrace{\left(1+k^{2}\right)^{r-s} 4 k^{4} e^{-2 k^{2} t}}_{\mathcal{G}(t, r):=}|\hat{\phi}(k)|^{2}\left(1+|k|^{2}\right)^{s}
\end{aligned}
$$

y como $|\mathcal{G}(t, r)| \leq C \forall(t, r) \in[\epsilon,+\infty) \times \mathbb{R}, \mathrm{y} \forall k \in Z$ donde $\epsilon$ es suficientemente pequeño, y por otro lado sabemos por hipótesis que la serie:

$$
2 \pi \sum_{k=-\infty}^{+\infty}\left(1+k^{2}\right)^{s}|\hat{\phi}(k)|^{2}=\|\phi\|_{H_{p e r}^{s}}^{2}<\infty
$$

desde que $\phi \in H_{p e r}^{s}$. Así, usando el Teorema M-Test de Weierstrass tenemos que la serie (4.1) converge uniformemente y por lo tanto es posible intercambiar límites y obtener lo que se quería mostrar, i.e.

$$
\left\|\frac{u(t+h)-u(t)}{h}-\partial_{x}^{2} u(t)\right\|_{H_{p e r}^{r}}^{2} \longrightarrow 0 \quad \text { cuando } h \rightarrow 0 .
$$

Teorema 5. Sea $s \in \mathbb{R}$. Si u es solución de $\left(P_{1}\right)$, entonces

$$
\lim _{h \rightarrow 0^{+}}\left\|\frac{u(h)-\phi}{h}-\partial_{x}^{2} u(0)\right\|_{r}=0, \forall r \leq s-2 .
$$

Esto es, $\partial_{t^{+}} u(0)=\partial_{x}^{2} u(0)$ en $H_{p e r}^{r}, \forall r \leq s-2$.

Demostración:

$$
\begin{aligned}
& \left\|\frac{u(h)-u(0)}{h}-\partial_{x}^{2} \phi\right\|_{r}^{2} \\
& \quad=\left.2 \pi \sum_{k=-\infty}^{+\infty}\left(1+k^{2}\right)^{r}|\underbrace{\frac{e^{-k^{2} h}-1}{h}+k^{2}}_{M(h):=}|^{2} \widehat{\phi}(k)\right|^{2} .
\end{aligned}
$$


Usando L'Hospital tenemos que $M(h) \longrightarrow 0$ cuando $h \rightarrow 0^{+}$.

Ahora, necesitamos la convergencia uniforme de la serie para habilitar el intercambio de límites. Para ello procedemos mayorando el k-ésimo término de la serie (4.4). Así, usando la estimativa (4.3) obtenemos

$$
\begin{aligned}
I_{k, r} & :=\left(1+k^{2}\right)^{r}|\hat{\phi}(k)|^{2}[M(h)]^{2} \\
& \leq\left(1+k^{2}\right)^{r+2} 4|\hat{\phi}(k)|^{2} .
\end{aligned}
$$

Por otro lado, como $\phi \in H_{p e r}^{s}$, tenemos que converge la serie:

$$
2 \pi \sum_{k=-\infty}^{+\infty}\left(1+k^{2}\right)^{s}|\hat{\phi}(k)|^{2}=\|\phi\|_{H_{p e r}^{s}}^{2}<\infty .
$$

Luego, usando la inmersion continua en los espacios de Sobolev periódico, i.e. $H_{\text {per }}^{s} \subset H_{\text {per }}^{r+2}$, para $r \leq s-2$, obtenemos

$$
8 \pi \sum_{k=-\infty}^{+\infty}\left(1+k^{2}\right)^{r+2}|\hat{\phi}(k)|^{2}=4\|\phi\|_{H_{p e r}^{r+2}}^{2} \leq 4\|\phi\|_{H_{p e r}^{s}}^{2}<\infty, \forall r \leq s-2 .
$$

Así, usando el Teorema M-Test de Weierstrass tenemos que la serie (4.4) converge uniformemente y por lo tanto es posible intercambiar límites y obtener lo que se quería mostrar, i.e.

$$
\left\|\frac{u(h)-u(0)}{h}-\partial_{x}^{2} u(0)\right\|_{H_{p e r}^{r}}^{2} \longrightarrow 0 \quad \text { cuando } h \rightarrow 0^{+} .
$$

Teorema 6. Sea $s \in \mathbb{R}$ y $\phi \in H_{\text {per }}^{s}$, son equivalentes los siguientes enunciados:

1. Existe $\lim _{h \rightarrow 0^{+}}\left(\frac{S(h)-1}{h}\right) \phi$ en $\left(H_{\text {per }}^{s},\|\cdot\|_{s}\right)$.

2. $\phi \in H_{\text {per }}^{s+2}$.

Demostración: Supongamos que se tenga el item 1, entonces $A=\partial_{x}^{2}$ es el generador infinitesimal del semigrupo de contracción $\{S(t)\}_{t \geq 0}$. Así, $A \phi=\partial_{x}^{2} \phi \in H_{p e r}^{s}$, de ahí tenemos

$$
\left(1-\partial_{x}^{2}\right) \phi=\phi-\partial_{x}^{2} \phi \in H_{p e r}^{s} .
$$

Usando la transformada de Fourier a (4.5) obtenemos

$$
\left(\left(1+k^{2}\right) \widehat{\phi}(k)\right)_{k \in Z} \in l_{s}^{2},
$$

de donde conseguimos

$$
\begin{aligned}
\|\phi\|_{s+2}^{2} & =2 \pi \sum_{k=-\infty}^{+\infty}\left(1+k^{2}\right)^{s+2}|\widehat{\phi}(k)|^{2} \\
& =2 \pi \sum_{k=-\infty}^{+\infty}\left(1+k^{2}\right)^{s}\left|\left(1+k^{2}\right) \widehat{\phi}(k)\right|^{2}<\infty .
\end{aligned}
$$

Entonces, $\phi \in H_{\text {per }}^{s+2}$.

Recíprocamente, sea $\phi \in H_{\text {per }}^{s+2}$,

$$
\left\|\frac{S(h) \phi-\phi}{h}-\partial_{x}^{2} \phi\right\|_{s}^{2}=2 \pi \sum_{k=-\infty}^{+\infty}\left(1+k^{2}\right)^{s}|\underbrace{\left(\frac{e^{-h k^{2}}-1}{h}\right)+k^{2}}_{M(h):=}|^{2}|\widehat{\phi}(k)|^{2} .
$$

Usando L'Hospital tenemos que $M(h) \longrightarrow 0$ cuando $h \rightarrow 0^{+}$.

Ahora, necesitamos la convergencia uniforme de la serie para habilitar el intercambio de límites. Para ello procedemos mayorando el k-ésimo término de la serie.

Previamente observamos para $h>0$ :

$$
\frac{e^{-k^{2} h}-1}{h}=\int_{0}^{h} \frac{1}{h} \frac{\partial}{\partial s}\left\{e^{-k^{2} s}\right\} d s=\int_{0}^{h} \frac{1}{h}\left[-k^{2}\right] e^{-k^{2} s} d s
$$


y tomando norma tenemos

$$
\left|\frac{e^{-k^{2} h}-1}{h}\right| \leq \frac{1}{h} k^{2} \int_{0}^{h}\left|e^{-k^{2} s}\right| d s \leq \frac{1}{h}|k|^{2} \cdot h=|k|^{2} .
$$

O también se puede proceder usando el Teorema del valor medio, aplicado a la función $f(t)=e^{-t k^{2}}$ en el intervalo $[0, h]$.

Usando la desigualdad (4.8) procedemos a mayorar $[M(h)]^{2}$ como sigue

$$
[M(h)]^{2} \leq\left\{2|k|^{2}\right\}^{2} \leq 4\left\{1+|k|^{2}\right\}^{2} .
$$

Pasamos a mayorar el k-ésimo término de la serie, donde se usa la estimativa (4.9)

$$
\begin{aligned}
\left(1+k^{2}\right)^{s}|\hat{\phi}(k)|^{2}[M(h)]^{2} & \leq\left(1+k^{2}\right)^{s}|\hat{\phi}(k)|^{2} 4\left(1+|k|^{2}\right)^{2} \\
& =4\left(1+k^{2}\right)^{s+2}|\hat{\phi}(k)|^{2}
\end{aligned}
$$

y sabemos que la serie $2 \pi \sum_{k=-\infty}^{+\infty}\left(1+k^{2}\right)^{s+2}|\hat{\phi}(k)|^{2}=\|\phi\|_{H_{p e r}^{s+2}}^{2}<\infty$ desde que $\phi \in H_{p e r}^{s+2}$. Usando el Teorema M-Test de Weierstrass tenemos que la serie (4.7) converge uniformemente y por lo tanto es posible intercambiar límites y obtener lo que se quería mostrar, i.e.

$$
\left\|\frac{u(h)-u(0)}{h}-\partial_{x}^{2} u(0)\right\|_{H_{p e r}^{s}}^{2} \longrightarrow 0 \quad \text { cuando } h \rightarrow 0^{+} .
$$

5. Ecuación del Calor no Homogénea. Estudiaremos la existencia de solución de la ecuación del calor no homogénea en espacios de Sobolev periódico.

Teorema 7. Sea $s \in \mathbb{R}$ fijado, $F \in C\left([0, T], H_{\text {per }}^{s}\right),\{S(t)\}_{t \geq 0}$ el semigrupo de contracción de clase $C_{0}$ definido en Teorema 2 y $u_{p}(t):=\int_{0}^{t} S(t-\tau) F(\tau) d \tau$. Entonces

$$
u_{p} \in C\left([0, T], H_{p e r}^{s}\right) \cap C^{1}\left([0, T], H_{p e r}^{s-2}\right)
$$

o mejor aún:

$$
u_{p} \in C\left([0, T], H_{p e r}^{r}\right) \cap C^{1}\left([0, T], H_{p e r}^{r-2}\right), \forall r \leq s .
$$

Consiguiendose obtener en $H_{p e r}^{s-2}$ :

$$
\partial_{t} u_{p}(t)=F(t)+\underbrace{\int_{0}^{t} \partial_{t} S(t-\tau) F(\tau) d \tau}_{=\partial_{x}^{2} u_{p}(t)}
$$

esto es, con respecto a la norma de $H_{p e r}^{s-2}$.

Mejor aún, $\partial_{t} u_{p}(t)=F(t)+\partial_{x}^{2} u_{p}(t)$ con respecto a la norma de $H_{p e r}^{r-2}, \forall r \leq s$.

Así, $u_{p}(t)$ satisface

$$
\left(P_{2, p}\right) \mid \begin{aligned}
& u_{p} \in C\left([0, T], H_{p e r}^{s}\right) \cap C^{1}\left([0, T], H_{p e r}^{s-2}\right) \\
& \partial_{t} u_{p}(t)-\partial_{x}^{2} u_{p}(t)=F(t) \in H_{p e r}^{s-2} \\
& u_{p}(0)=0
\end{aligned}
$$

con la primera derivada calculada en la norma de $H_{p e r}^{s-2}$.

Demostración:Probaremos que $u_{p}$ es continua. En efecto, para $t<t^{\prime}$ y $\tau \in\left(t, t^{\prime}\right)$,

$$
\begin{aligned}
& \left\|u_{p}(t)-u_{p}\left(t^{\prime}\right)\right\|_{s} \\
& \quad=\left\|\int_{0}^{t} S(t-\tau) F(\tau) d \tau-\int_{0}^{t^{\prime}} S\left(t^{\prime}-\tau\right) F(\tau) d \tau\right\|_{s} \\
& \quad \leq\left\|\int_{0}^{t}\left\{S(t-\tau)-S\left(t^{\prime}-\tau\right)\right\} F(\tau) d \tau\right\|_{s}+\left\|\int_{t}^{t^{\prime}} S\left(t^{\prime}-\tau\right) F(\tau) d \tau\right\|_{s} \\
& \quad \leq \int_{0}^{t}\left\|\left\{S(t-\tau)-S\left(t^{\prime}-\tau\right)\right\} F(\tau)\right\|_{s} d \tau+\int_{t}^{t^{\prime}}\left\|S\left(t^{\prime}-\tau\right) F(\tau)\right\|_{s} d \tau .
\end{aligned}
$$


Por otro lado, conseguimos:

$$
\int_{0}^{t}\left\|\left\{S(t-\tau)-S\left(t^{\prime}-\tau\right)\right\} F(\tau)\right\|_{s} d \tau<\epsilon \int_{0}^{t} d \tau=\epsilon t \leq \epsilon T,
$$

siempre que $\left|t-t^{\prime}\right|<\delta$.

También, obtenemos:

$$
\begin{aligned}
\int_{t}^{t^{\prime}}\left\|S\left(t^{\prime}-\tau\right) F(\tau)\right\|_{s} d \tau & \leq \int_{t}^{t^{\prime}}\|F(\tau)\|_{s} d \tau \\
& \leq \sup _{\tau \in[0, T]}\|F(\tau)\|_{s} \int_{t}^{t^{\prime}} d \tau \\
& =\left(t^{\prime}-t\right) \sup _{\tau \in[0, T]}\|F(\tau)\|_{s}
\end{aligned}
$$

Usando (5.2) y (5.3) en (5.1) obtenemos

$$
\lim _{t \rightarrow t^{\prime}}\left\|u_{p}(t)-u_{p}\left(t^{\prime}\right)\right\|_{s}=0 .
$$

Análogamente probamos que

$$
\lim _{t \rightarrow t^{\prime}}\left\|u_{p}(t)-u_{p}\left(t^{\prime}\right)\right\|_{r}=0, \forall r \leq s .
$$

En segundo lugar, usando la versión generalizada al cálculo diferencial en espacios de Banach, obtenemos en $H_{p e r}^{s-2}$ :

$$
\begin{aligned}
\partial_{t} u_{p}(t) & =\underbrace{S(t-t) F(t)}_{=F(t)}-S(t-0) F(0) \cdot 0+\int_{0}^{t} \partial_{t} S(t-\tau) F(\tau) d \tau \\
& =F(t)+\int_{0}^{t} \partial_{t} S(t-\tau) F(\tau) d \tau=F(t)+\partial_{x}^{2} u_{p}(t) .
\end{aligned}
$$

Similarmente, vale $\partial_{t} u_{p}(t)=F(t)+\int_{0}^{t} \partial_{t} S(t-\tau) F(\tau) d \tau=F(t)+\partial_{x}^{2} u_{p}(t)$ en $H_{p e r}^{r-2}, \forall r \leq s$. Ahora, probaremos que $\partial_{t} u_{p}$ es continua. Para $t<t^{\prime}$ y $\tau \in\left(t, t^{\prime}\right)$,

$$
\begin{aligned}
\left\|\partial_{t} u_{p}(t)-\partial_{t} u_{p}\left(t^{\prime}\right)\right\|_{s-2} & =\left\|F(t)+\partial_{x}^{2} u_{p}(t)-\left\{F\left(t^{\prime}\right)+\partial_{x}^{2} u_{p}\left(t^{\prime}\right)\right\}\right\|_{s-2} \\
& \leq\left\|F(t)-F\left(t^{\prime}\right)\right\|_{s-2}+\left\|\partial_{x}^{2} u_{p}(t)-\partial_{x}^{2} u_{p}\left(t^{\prime}\right)\right\|_{s-2} \\
& \leq\left\|F(t)-F\left(t^{\prime}\right)\right\|_{s}+\left\|u_{p}(t)-u_{p}\left(t^{\prime}\right)\right\|_{s} .
\end{aligned}
$$

Usando la continuidad de $F$ y $u_{p}$ en (5.4) obtenemos que

$$
\lim _{t \rightarrow t^{\prime}}\left\|\partial_{t} u_{p}(t)-\partial_{t} u_{p}\left(t^{\prime}\right)\right\|_{s-2}=0 .
$$

Análogamente, probamos que $\operatorname{lím}_{t \rightarrow t^{\prime}}\left\|\partial_{t} u_{p}(t)-\partial_{t} u_{p}\left(t^{\prime}\right)\right\|_{r-2}=0, \forall r \leq s$.

Finalmente, hemos obtenido en $H_{p e r}^{s-2}: \partial_{t} u_{p}(t)=F(t)+\partial_{x}^{2} u_{p}(t)$ y evidentemente $u_{p}(0)=0$.

Teorema 8 (Existencia de solución local). Sea $T>0, s \in \mathbb{R}$ fijado y $F \in C\left([0, T], H_{\text {per }}^{s}\right)$

$$
\begin{array}{l|l}
\left(P_{1}^{F}\right) & \begin{array}{l}
\partial_{t} u-\partial_{x}^{2} u=F(t) \in H_{p e r}^{s-2} \\
u(0)=\phi \in H_{p e r}^{s}
\end{array}
\end{array}
$$

entonces $\exists ! u \in C\left([0, T], H_{\text {per }}^{s}\right) \cap C^{1}\left([0, T], H_{\text {per }}^{s-2}\right)$ solución de $\left(P_{1}^{F}\right)$. Mejor aún, $u \in C\left([0, T], H_{\text {per }}^{r}\right) \cap C^{1}\left([0, T], H_{\text {per }}^{r-2}\right)$, $\forall r \leq s$.

Demostración:La prueba lo haremos del siguiente modo.

1. Primero, obtenemos el candidato a solución. Para conseguir esto, aplicamos la transformada de Fourier a la ecuación no homogénea $\left(P_{1}^{F}\right)$

$$
\partial_{t} u(t)-\partial_{x}^{2} u(t)=F(t)
$$

$\mathrm{y}$ tenemos

$$
\partial_{t} \widehat{u}(k, t)=-k^{2} \widehat{u}(k, t)+\widehat{F}(k, t)
$$


que para cada $k$ es una EDO no homogénea con dato inicial $\widehat{u}(k, 0)=\widehat{\phi}(k)$.

Así, planteamos un sistema no acoplado de ecuaciones de primer orden no homogéneo

$$
\begin{array}{l|l}
\left(\Omega_{k}\right) & \begin{array}{l}
\widehat{u} \in C\left([0, T], l_{s}^{2}(Z)\right) \\
\partial_{t} \widehat{u}(k, t)=-k^{2} \widehat{u}(k, t)+\widehat{F}(k, t) \\
\widehat{u}(k, 0)=\widehat{\phi}(k) \operatorname{con} \widehat{\phi} \in l_{s}^{2}(Z),
\end{array}
\end{array}
$$

$\forall k \in Z$, que resolveremos a continuación.

Sea $k \neq 0$, multiplicando por el factor integrante $e^{k^{2} t}$, a la ecuación diferencial de $\left(\Omega_{k}\right)$, obtenemos

$$
\partial_{t}\left\{e^{k^{2} t} \widehat{u}(k, t)\right\}=e^{k^{2} t} \widehat{F}(k, t),
$$

integrando de 0 a $t$, obtenemos

$$
\int_{0}^{t} \partial_{t}\left\{e^{k^{2} \tau} \widehat{u}(k, \tau)\right\} d \tau=\int_{0}^{t} e^{k^{2} \tau} \widehat{F}(k, \tau) d \tau
$$

luego,

$$
e^{k^{2}} \widehat{u}(k, t)-\widehat{u}(k, 0)=\int_{0}^{t} e^{k^{2} \tau} \widehat{F}(k, \tau) d \tau
$$

esto es,

$$
\begin{aligned}
\widehat{u}(k, t) & =e^{-k^{2} t} \widehat{\phi}(k)+e^{-k^{2} t} \int_{0}^{t} e^{k^{2} \tau} \widehat{F}(k, \tau) d \tau \\
& =e^{-k^{2} t} \widehat{\phi}(k)+\int_{0}^{t} e^{-k^{2}(t-\tau)} \widehat{F}(k, \tau) d \tau .
\end{aligned}
$$

Si $k=0$, la EDO no homogénea es:

$$
\mid \begin{aligned}
& \partial_{t} \widehat{u}(0, t)=\widehat{F}(0, t) \\
& \widehat{u}(0,0)=\widehat{\phi}(0)
\end{aligned}
$$

Integrando de 0 a $t$, obtenemos

$$
\int_{0}^{t} \partial_{t} \widehat{u}(0, \tau) d \tau=\int_{0}^{t} \widehat{F}(0, \tau) d \tau
$$

luego

$$
\widehat{u}(0, t)-\widehat{u}(0,0)=\int_{0}^{t} \widehat{F}(0, \tau) d \tau,
$$

esto es,

$$
\widehat{u}(0, t)=\widehat{\phi}(0)+\int_{0}^{t} \widehat{F}(0, \tau) d \tau .
$$

Finalmente,

$$
\widehat{u}(k, t)=e^{-k^{2} t} \widehat{\phi}(k)+\int_{0}^{t} e^{-k^{2}(t-\tau)} \widehat{F}(k, \tau) d \tau, \forall k \in Z .
$$

El candidato a solución de $\left(P_{1}^{F}\right)$ es

$$
\begin{aligned}
u(t) & =\sum_{k=-\infty}^{+\infty} \widehat{u}(k, t) \phi_{k} \\
& =\sum_{k=-\infty}^{+\infty}\left\{e^{-k^{2} t} \widehat{\phi}(k)+\int_{0}^{t} e^{-k^{2}(t-\tau)} \widehat{F}(k, \tau) d \tau\right\} \phi_{k} \\
& =\sum_{k=-\infty}^{+\infty} e^{-k^{2} t} \widehat{\phi}(k) \phi_{k}+\sum_{k=-\infty}^{+\infty}\left\{\int_{0}^{t} e^{-k^{2}(t-\tau)} \widehat{F}(k, \tau) d \tau\right\} \phi_{k} \\
& =\sum_{k=-\infty}^{+\infty} e^{-k^{2} t} \widehat{\phi}(k) \phi_{k}+\int_{0}^{t} \sum_{k=-\infty}^{+\infty} e^{-k^{2}(t-\tau)} \widehat{F}(k, \tau) \phi_{k} d \tau \\
& =\underbrace{S(t) \phi}_{u_{h}(t):=}+\underbrace{\int_{0}^{t} S(t-\tau) F(\tau) d \tau}_{u_{p}(t):=},
\end{aligned}
$$


donde $u_{h}$ es la solución de la ecuación homogénea de $\left(P_{1}^{F}\right)$ que ya fue probada y $u_{p}$ es la solución particular de $\left(P_{1}^{F}\right)$ con condición nula, que también fue probada en el teorema previo.

2. Observamos que $u(t) \in H_{p e r}^{s}$. Además, como $u_{h}, u_{p} \in C\left([0, T], H_{p e r}^{s}\right)$, entonces $u=u_{h}+u_{p} \in C\left([0, T], H_{p e r}^{s}\right)$. Similarmente, como $u_{h}, u_{p} \in C^{1}\left([0, T], H_{\text {per }}^{s-2}\right)$, entonces $u=u_{h}+u_{p} \in C^{1}\left([0, T], H_{\text {per }}^{s-2}\right)$.

Similarmete se prueba que $u=u_{h}+u_{p} \in C\left([0, T], H_{\text {per }}^{r}\right) \cap C^{1}\left([0, T], H_{\text {per }}^{r-2}\right), \forall r \leq s$.

También, verificamos que $u(0)=u_{h}(0)+u_{p}(0)=\phi+0=\phi$.

Como existen $\partial_{t} u_{h}(t), \partial_{t} u_{p}(t)$ en $H_{p e r}^{s-2}$, entonces $u(t)=u_{h}(t)+u_{p}(t)$ satisface en $H_{p e r}^{s-2}$ :

$$
\begin{aligned}
\partial_{t} u(t) & =\partial_{t} u_{h}(t)+\partial_{t} u_{p}(t) \\
& =\partial_{x}^{2} u_{h}(t)+\left(\partial_{x}^{2} u_{p}(t)+F(t)\right) \\
& =\left(\partial_{x}^{2} u_{h}(t)+\partial_{x}^{2} u_{p}(t)\right)+F(t) \\
& =\partial_{x}^{2}\left(u_{h}(t)+u_{p}(t)\right)+F(t) \\
& =\partial_{x}^{2} u(t)+F(t) .
\end{aligned}
$$

Corolario 3. La única solución de $\left(P_{1}^{F}\right)$ es

$$
u(x, t)=\sum_{k=-\infty}^{+\infty} e^{-k^{2} t} \widehat{\phi}(k) e^{i k x}+\int_{0}^{t} \sum_{k=-\infty}^{+\infty} e^{-k^{2}(t-\tau)} \widehat{F}(k, \tau) e^{i k x} d \tau
$$

Teorema 9. Sea $T>0$, la solución de $\left(P_{1}^{F}\right)$ satisface

$$
\begin{array}{r}
\|u(t)\|_{r} \leq\|\phi\|_{s}+T\|F\|_{s, \infty}, \forall r \leq s, \\
\sup _{t \in[0, T]}\|u(t)\|_{r} \leq\|\phi\|_{s}+T\|F\|_{s, \infty}, \forall r \leq s .
\end{array}
$$

Además

$$
\begin{aligned}
\left\|\partial_{t} u(t)\right\|_{r-2} & \leq\|\phi\|_{s}+(1+T)\|F\|_{s, \infty}, \\
\sup _{t \in[0, T]}\left\|\partial_{t} u(t)\right\|_{r-2} & \leq\|\phi\|_{s}+(1+T)\|F\|_{s, \infty}
\end{aligned}
$$

$\forall r \leq s$, donde $\|F\|_{s, \infty}:=\sup _{t \in[0, T]}\|F(t)\|_{s}$

Demostración: Sea $u$ solución de $\left(P_{1}^{F}\right)$ entonces

$$
u(t)=S(t) \phi+\int_{0}^{t} S(t-\tau) F(\tau) d \tau .
$$

Usando la desigualdad triangular de la norma $\|\cdot\|_{r}$, inmersión continua de $H_{\text {per }}^{s}$ en $H_{\text {per }}^{r} \forall r \leq s,\|S(t) \psi\|_{s} \leq\|\psi\|_{s}$ para $\psi \in H_{\text {per }}^{s}$, obtenemos

$$
\begin{aligned}
\|u(t)\|_{r} & \leq\left\|S(t) \phi+\int_{0}^{t} S(t-\tau) F(\tau) d \tau\right\|_{r} \\
& \leq\|S(t) \phi\|_{r}+\left\|\int_{0}^{t} S(t-\tau) F(\tau) d \tau\right\|_{r} \\
& \leq\|S(t) \phi\|_{s}+\left\|\int_{0}^{t} S(t-\tau) F(\tau) d \tau\right\|_{s} \\
& \leq\|\phi\|_{s}+\int_{0}^{t}\|S(t-\tau) F(\tau)\|_{s} d \tau \\
& \leq\|\phi\|_{s}+\int_{0}^{t}\|F(\tau)\|_{s} d \tau \\
& \leq\|\phi\|_{s}+\sup _{\tau \in[0, T]}\|F(\tau)\|_{s} \int_{0}^{t} d \tau \\
& \leq\|\phi\|_{s}+T\|F\|_{s, \infty}, \forall r \leq s .
\end{aligned}
$$

Tomando supremo en la desigualdad (5.5) tenemos

$$
\sup _{t \in[0, T]}\|u(t)\|_{r} \leq\|\phi\|_{s}+T\|F\|_{s, \infty}, \forall r \leq s .
$$


Por otro lado, vale en $H_{p e r}^{s-2}$ la igualdad $\partial_{t} u(t)=\partial_{x}^{2} u(t)+F(t)$.

Usando la desigualdad triangular de la norma $\|\cdot\|_{r-2}$, la inmersión continua de $H_{p e r}^{s-2} \subset H_{p e r}^{r-2} \forall r \leq s$ y que el operador diferencial $\partial_{x}^{2}$ va de $H_{p e r}^{s}$ en $H_{p e r}^{s-2}$, obtenemos

$$
\begin{aligned}
\left\|\partial_{t} u(t)\right\|_{r-2} & =\left\|\partial_{x}^{2} u(t)+F(t)\right\|_{r-2} \\
& \leq\left\|\partial_{x}^{2} u(t)\right\|_{r-2}+\|F(t)\|_{r-2} \\
& \leq\left\|\partial_{x}^{2} u(t)\right\|_{s-2}+\|F(t)\|_{s-2} \\
& \leq\|u(t)\|_{s}+\|F(t)\|_{s}, \quad \forall r \leq s .
\end{aligned}
$$

Usando (5.5) en (5.6) obtenemos

$$
\begin{aligned}
\left\|\partial_{t} u(t)\right\|_{r} & \leq\|\phi\|_{s}+T\|F\|_{s, \infty}+\|F(t)\|_{s} \\
& \leq\|\phi\|_{s}+(T+1)\|F\|_{s, \infty}, \quad \forall r \leq s .
\end{aligned}
$$

Tomando supremo en la desigualdad (5.7) ontenemos

$$
\sup _{t \in[0, T]}\left\|\partial_{t} u(t)\right\|_{r-2} \leq\|\phi\|_{s}+(T+1)\|F\|_{s, \infty}, \quad \forall r \leq s .
$$

A continuación enunciaremos y probaremos la dependencia continua de la solución de $\left(P_{1}^{F}\right)$ respecto al dato inicial y a la no homogeneidad $F$.

Teorema 10. (Dependencia continua de la solución respecto al dato inicial y a la no homogeneidad) Sea $T>0$, s un número real fijado, $\varphi_{j} \in H_{p e r}^{s}, F_{j} \in C\left([0, T], H_{p e r}^{s}\right)$ y denotemos por $u_{j}$ a la correspondiente solución de $\left(P_{1}^{F_{j}}\right)$ para $j=1,2$. Entonces

$$
\begin{aligned}
\left\|u_{1}(t)-u_{2}(t)\right\|_{s} & \leq\left\|\varphi_{1}-\varphi_{2}\right\|_{s}+T\left\|F_{1}-F_{2}\right\|_{s, \infty}, \\
\sup _{t \in[0, T]}\left\|u_{1}(t)-u_{2}(t)\right\|_{s} & \leq\left\|\varphi_{1}-\varphi_{2}\right\|_{s}+T\left\|F_{1}-F_{2}\right\|_{s, \infty}
\end{aligned}
$$

$y$

$$
\begin{aligned}
\left\|\partial_{t} u_{1}(t)-\partial_{t} u_{2}(t)\right\|_{s-2} & \leq\left\|\varphi_{1}-\varphi_{2}\right\|_{s}+(1+T)\left\|F_{1}-F_{2}\right\|_{s, \infty} \\
\sup _{t \in[0, T]}\left\|\partial_{t} u_{1}(t)-\partial_{t} u_{2}(t)\right\|_{s-2} & \leq\left\|\varphi_{1}-\varphi_{2}\right\|_{s}+(1+T)\left\|F_{1}-F_{2}\right\|_{s, \infty}
\end{aligned}
$$

donde $\|F\|_{s, \infty}:=\sup _{\tau \in[0, T]}\|F(\tau)\|_{s}$.

O mejor aún, $\forall r \leq s$

$$
\begin{aligned}
\left\|u_{1}(t)-u_{2}(t)\right\|_{r} & \leq\left\|\varphi_{1}-\varphi_{2}\right\|_{s}+T\left\|F_{1}-F_{2}\right\|_{s, \infty} \\
\sup _{t \in[0, T]}\left\|u_{1}(t)-u_{2}(t)\right\|_{r} & \leq\left\|\varphi_{1}-\varphi_{2}\right\|_{s}+T\left\|F_{1}-F_{2}\right\|_{s, \infty}
\end{aligned}
$$

$y$

$$
\begin{aligned}
\left\|\partial_{t} u_{1}(t)-\partial_{t} u_{2}(t)\right\|_{r-2} & \leq\left\|\varphi_{1}-\varphi_{2}\right\|_{s}+(1+T)\left\|F_{1}-F_{2}\right\|_{s, \infty}, \\
\sup _{t \in[0, T]}\left\|\partial_{t} u_{1}(t)-\partial_{t} u_{2}(t)\right\|_{r-2} & \leq\left\|\varphi_{1}-\varphi_{2}\right\|_{s}+(1+T)\left\|F_{1}-F_{2}\right\|_{s, \infty} .
\end{aligned}
$$

Demostración:Sea $u_{i}$ solución de $\left(P_{1}^{F_{i}}\right)$ entonces

$$
u_{i}(t)=\underbrace{S(t) \varphi_{i}}_{u_{i, h}(t):=}+\underbrace{\int_{0}^{t} S(t-\tau) F_{i}(\tau) d \tau}_{u_{i, p}(t):=}
$$

para $i=1,2$.

Tomando la diferencia de $u_{1}(t)$ con $u_{2}(t)$, tenemos en $H_{p e r}^{s}$ :

$$
u_{1}(t)-u_{2}(t)=S(t)\left\{\varphi_{1}-\varphi_{2}\right\}+\int_{0}^{t} S(t-\tau)\left\{F_{1}(\tau)-F_{2}(\tau)\right\} d \tau
$$


Ahora, usando la desigualdad triangular de la norma $\|\cdot\|_{r}$, inmersión continua de $H_{\text {per }}^{s}$ en $H_{\text {per }}^{r}$ para $r \leq s$ y $\|S(t) \psi\|_{s} \leq\|\psi\|_{s}$ con $\psi \in H_{p e r}^{s}$, obtenemos

$$
\begin{aligned}
\left\|u_{1}(t)-u_{2}(t)\right\|_{r} & \leq\left\|S(t)\left\{\varphi_{1}-\varphi_{2}\right\}\right\|_{r}+\left\|\int_{0}^{t} S(t-\tau)\left\{F_{1}(\tau)-F_{2}(\tau)\right\} d \tau\right\|_{r} \\
& \leq\left\|S(t)\left\{\varphi_{1}-\varphi_{2}\right\}\right\|_{s}+\int_{0}^{t}\left\|S(t-\tau)\left\{F_{1}(\tau)-F_{2}(\tau)\right\}\right\|_{r} d \tau \\
& \leq\left\|\varphi_{1}-\varphi_{2}\right\|_{s}+\int_{0}^{t}\left\|S(t-\tau)\left\{F_{1}(\tau)-F_{2}(\tau)\right\}\right\|_{s} d \tau \\
& \leq\left\|\varphi_{1}-\varphi_{2}\right\|_{s}+\int_{0}^{t}\left\|F_{1}(\tau)-F_{2}(\tau)\right\|_{s} d \tau \\
& \leq\left\|\varphi_{1}-\varphi_{2}\right\|_{s}+\sup _{\tau \in[0, T]}\left\|F_{1}(\tau)-F_{2}(\tau)\right\|_{s} \int_{0}^{t} d \tau \\
& \leq\left\|\varphi_{1}-\varphi_{2}\right\|_{s}+T \sup _{\tau \in[0, T]}\left\|F_{1}(\tau)-F_{2}(\tau)\right\|_{s}, \forall r \leq s .
\end{aligned}
$$

Tomando supremo en la desigualdad (5.8) obtenemos

$$
\sup _{t \in[0, T]}\left\|u_{1}(t)-u_{2}(t)\right\|_{r} \leq\left\|\varphi_{1}-\varphi_{2}\right\|_{s}+T\left\|F_{1}-F_{2}\right\|_{s, \infty}, \quad \forall r \leq s
$$

Por otro lado, en $H_{\text {per }}^{s-2}$ para $i=1,2$, tenemos

$$
\partial_{t} u_{i}(t)=F_{i}(t)+\partial_{x}^{2} u_{i}(t)
$$

Tomando la diferencia de $\partial_{t} u_{1}(t)$ con $\partial_{t} u_{2}(t)$ tenemos en $H_{p e r}^{s-2}$ :

$$
\partial_{t} u_{1}(t)-\partial_{t} u_{2}(t)=F_{1}(t)-F_{2}(t)+\partial_{x}^{2} u_{1}(t)-\partial_{x}^{2} u_{2}(t)
$$

y usando la desigualdad triangular de la norma $\|\cdot\|_{r-2}$, la inmersión continua de $H_{p e r}^{s-2} \subset H_{p e r}^{r-2} \forall r \leq s$ y que el operador diferencial $\partial_{x}^{2}$ va de $H_{p e r}^{s}$ en $H_{p e r}^{s-2}$

$$
\begin{aligned}
\left\|\partial_{t} u_{1}(t)-\partial_{t} u_{2}(t)\right\|_{r-2} & \leq\left\|F_{1}(t)-F_{2}(t)\right\|_{r-2}+\left\|\partial_{x}^{2}\left\{u_{1}(t)-u_{2}(t)\right\}\right\|_{r-2} \\
& \leq\left\|F_{1}(t)-F_{2}(t)\right\|_{s-2}+\left\|\partial_{x}^{2}\left\{u_{1}(t)-u_{2}(t)\right\}\right\|_{s-2} \\
& \leq\left\|F_{1}(t)-F_{2}(t)\right\|_{s}+\left\|u_{1}(t)-u_{2}(t)\right\|_{s} .
\end{aligned}
$$

Usando (5.8) en (5.9), obtenemos para $r \leq s$ :

$$
\begin{aligned}
\left\|\partial_{t} u_{1}(t)-\partial_{t} u_{2}(t)\right\|_{r-2} & \leq\left\|F_{1}(t)-F_{2}(t)\right\|_{s}+\left\|\varphi_{1}-\varphi_{2}\right\|_{s}+T\left\|F_{1}-F_{2}\right\|_{s, \infty} \\
& \leq\left\|\varphi_{1}-\varphi_{2}\right\|_{s}+(1+T)\left\|F_{1}-F_{2}\right\|_{s, \infty}
\end{aligned}
$$

Tomando supremo en la desigualdad (5.10) obtenemos

$$
\sup _{t \in[0, T]}\left\|\partial_{t} u_{1}(t)-\partial_{t} u_{2}(t)\right\|_{r-2} \leq\left\|\varphi_{1}-\varphi_{2}\right\|_{s}+(1+T)\left\|F_{1}-F_{2}\right\|_{s, \infty}
$$

$\forall r \leq s$

6. Conclusiones. En nuestro estudio de la ecuación del calor en espacios de Sobolev periódico tanto en el caso homogéneo $\left(P_{1}\right)$ como en el correspondiente problema no homogéneo $\left(P_{1}^{F}\right)$ hemos obtenido importantes resultados, entre los cuales destacamos:

1. Usando la teoría de Fourier, demostramos la existencia y unicidad de solución del modelo $\left(P_{1}\right)$, así como la dependencia continua de la solución respecto al dato inicial.

2. Probamos la regularidad de la solución de $\left(P_{1}\right)$.

3. Introduciendo una familia de operadores, la cual forma un $C_{0}$-Semigrupo, reescribimos la solución del problema $\left(P_{1}\right)$, obteniendo resultados más elegantes.

4. En el análisis de diferenciabilidad de la solución versus el dato inicial obtenemos resultados como el saber en qué espacio $H_{\text {per }}^{r}$ existe la derivada $\partial_{t} u(t)=\partial_{x}^{2}(t)$ y que esto depende mucho del espacio donde se tome el dato inicial. 
5. Usando la teoría de Fourier y la teoría de Semigrupo probamos la existencia de solución local y unicidad de solución del modelo no homogéneo $\left(P_{1}^{F}\right)$.

6. También, obtenemos la dependencia continua de la solución de $\left(P_{1}^{F}\right)$ respecto al dato inicial y a la parte no homogenea del problema.

Referencias

[1] Iorio R. and Iorio V., Fourier Analysis and partial differential equation. Cambridge University, 2001.

[2] Santiago Y. Rojas S., Quispe T., Espacios de Sobolev periódico y un problema de Cauchy asociado a un modelo de ondas en un fluido viscoso, Theorema, Segunda Época 3(4) (2016) 7-23.

[3] Santiago, Y. Rojas, S., Regularity and wellposedness of a problem to one parameter and its behavior at the limit, Bulletin of the Allahabad Mathematical Society 32(2) (2017) 207-230.

[4] Rubinstein, I. and Rubinstein, L., Partial differential equations in classical mathematical physics. Cambridge University Press, 1998.

[5] Shahjalal M., Sultana, A., Valluri R., Mitra, N. and Khan, A.; Black-Scholes PDE and Ornstein-Uhlenbeck SDE process to analyse stock option: A study in Fuzzy context, Intern. Journal of Mathematics and Computing 1(1) (2015) 1-10. 